\title{
Development of multidisciplinary nanotechnology undergraduate education program at the University of Rochester Integrated Nanosystems Center
}

Svetlana Lukishova, Nicholas Bigelow, Paul D'Alessandris

Svetlana G. Lukishova, Nicholas P. Bigelow, Paul D. D'Alessandris, "Development of multidisciplinary nanotechnology undergraduate education program at the University of Rochester Integrated Nanosystems Center," Proc. SPIE 10452, 14th Conference on Education and Training in Optics and Photonics: ETOP 2017, 1045210 (16 August 2017); doi: 10.1117/12.2269786

EDEent: 14th Conference on Education and Training in Optics and Photonics, ETOP 2017, 2017, Hangzhou, China 


\title{
Development of multidisciplinary nanotechnology undergraduate education program at the University of Rochester Integrated Nanosystems Center
}

\author{
Svetlana G. Lukishova**, Nicholas P. Bigelow ${ }^{\mathrm{a}, \mathrm{b}}$, Paul D. D’Alessandris ${ }^{\mathrm{c}}$

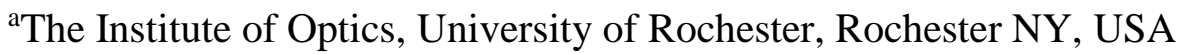 \\ ${ }^{b}$ Department of Physics and Astronomy, University of Rochester, Rochester NY, USA \\ ${ }^{\mathrm{c}}$ Monroe Community College, Rochester NY, USA \\ *E-mail: sluk@1le.rochester.edu
}

\begin{abstract}
Supported by the U.S. National Science Foundation educational grant, a coherent educational program at the University of Rochester (UR) in nanoscience and nanoengineering, based on the Institute of Optics and Intergrated Nanosystems Center resources was created. The main achievements of this program are (1) developing curriculum and offering the Certificate for Nanoscience and Nanoengineering program (15 students were awarded the Certificate and approximately 10 other students are working in this direction), (2) creating a reproducible model of collaboration in nanotechnology between a university with state-of-the-art, expensive experimental facilities, and a nearby, two-year community college (CC) with participation of a local Monroe Community College (MCC). 52 MCC students carried out two labs at the UR on the atomic force microscopy and a photolithography at a clean room; (3) developing reproducible hand-on experiments on nanophotonics ("mini-labs"), learning materials and pedagogical methods to educate students with diverse backgrounds, including freshmen and non-STEM-major CC students. These minilabs on nanophotonics were also introduced in some Institute of Optics classes. For the Certificate program UR students must take three courses: Nanometrology Laboratory (a new course) and two other selective courses from the list of several. Students also should carry out a one-semester research or a design project in the field of nanoscience and nanoengineering.
\end{abstract}

Keywords: Certificate for Nanoscience and Nanoengineering, Collaboration between the university and local community college, Mini-nano-labs, The Institute of Optics, University of Rochester, Monroe Community College

\section{INTRODUCTION}

We describe the University of Rochester (UR) successful project that addresses one of the most important concepts of modern engineering education geared towards increasingly important technological problems. Nanotechnology has the undisputed potential for creating many new materials and devices with wide-ranging applications. It is important to enable the future workforce to further develop these new ideas, as well as to provide students with hands-on experience in nanotechnology methods and tools for today's jobs. Modern students should be (1) fluent in nanoscience and nanotechnology terminology, (2) able to define nanoscience, nanotechnology, and nanodevices, (3) able to develop nanotechnology questions and propose strategies to answer them, (4) able to operate some nanotechnology characterization tools (STEM majors), and (5) choose a research career in nanoscience/nanotechnology (STEM majors).

Based on multi-million-dollar federal funding for equipment, the Integrated Nanosystems Center (URNano) opened at the University of Rochester in August 2011. This Center is truly interdisciplinary, and involves faculty and students from the Departments of Optics, Chemistry, Physics, Biomedical, Chemical, Mechanical and Electrical Engineering, as well as the UR Medical Center. Educational program of the URNano was developed in 2014-2017 years supported by the U.S. National Science Foundation (NSF) grant for the project "NUE: Development of Multidisciplinary Nanotechnology Undergraduate Education Program at the University of Rochester Integrated Nanosystems Center".

In this paper we describe our results on (a) creating a coherent educational program at the UR on the Certificate for Nanoscience and Nanoengineering (Section 2); (b) creating a transformable model of collaboration in nanotechnology between a university with state-of-the-art, expensive experimental facilities, and a nearby, two-year community college (CC) (Section 3); (c) developing reproducible hand-on experiments ("mini-labs"), learning materials and pedagogical methods to educate students with diverse backgrounds, including freshmen and non-STEM-major CC students (Section

14th Conference on Education and Training in Optics and Photonics: ETOP 2017, edited by Xu Liu,

Xi-Cheng Zhang, Proc. of SPIE Vol. 10452, 1045210 - (C) 2017 ICO, IEEE, OSA, SPIE

CCC code: $0277-786 X / 17 / \$ 18 \cdot$ doi: $10.1117 / 12.2269786$

Proc. of SPIE Vol. 10452 1045210-1 
4). The same "mini-labs" were introduced in various UR courses for UR freshmen and sophomores. Based on earlier NSF supported collaboration [1] between the UR and Monroe Community College (MCC), UR and MCC continued this collaboration to educate nanotechnology students with diverse backgrounds. Section 5 outlines some pedagogical methods for research in students' learning. Section 6 concludes the paper.

Below in this Introduction (Sections 1.1 and 1.2), some information is provided about URNano Center at the UR and Monroe Community College.

\subsection{The University of Rochester Integrated Nanosystems Center (URNano)}

The University of Rochester Integrated Nanosystems Center (URNano) was created by various U.S. government agencies' funding with grants totaling $\$ 4$ million. NY Congresswoman L. Slaughter aided efforts in obtaining these grants. Now the UR has a centralized nanofabrication facility linked with nanometrology instruments. Metrology (measurement) facility is located on the 1,000-square-foot area. The funding provided for a renovation of the electron microscopy facility, as well as the creation of a brand-new, 2,000-square-foot, the class 1000 clean room nanofabrication facility. The URNano houses scanning electron with focused ion beam, transmission electron, atomic force, and optical microscopes, deposition and etching equipment, and devices capable of lithography at nanoscales. The real-world applications being pursued at the URNano include new fuel cells, new dialysis techniques, and new devices for detecting ultra-small quantities of biological materials. URNano website address is https://www.rochester.edu/urnano/ . URnano also offers training for faculty, students, and outside researchers to become certified in the use of these tools. By making these tools accessible to a wide range of users, URnano trains current and future generations of technologists, furthers research in a variety of fields, and helps businesses translate that research into viable, innovative solutions.

\subsection{Monroe Community College (Rochester NY)}

Monroe Community College (MCC)'s Engineering Science/Physics program is a rigorous academic curriculum designed to facilitate transfer into B.S. Engineering/Physics programs ( https://www.monroecc.edu/depts/engineering-science-andphysics/). To date, this program's graduates have transferred into over 30 four-year institutions in NY State and nationally and MCC has 2+2 Dual Admission agreements with UR Biomedical, Chemical, Electrical and Mechanical Engineering, and Optics. In addition to STEM majors, non-STEM Liberal Arts majors were involved and benefited from the collaboration with the UR. Approximately $34 \%$ of MCC students are minorities and well over 50\% are women (non-Majors).

\section{UR PROGRAM ON CERTIFICATE IN NANOENGINEERING AND NANOSCIENCE FOR UNDERGRADUATE STUDENTS}

The program on a Certificate in Nanoengineering and Nanoscience for UR undergraduate students started in 2014 with 15 students awarded the Certificate by May 2017, has following requirements for its completion (see Figure 1):

(1) A required 4 credit hour laboratory course OPT 254/PHY 371 "Nanometrology Laboratory". This new course started in Spring 2015 was specially prepared for this program.

(2) On students' selection, two other courses containing nanotechnology content (see the list of some possible courses in a diagram of Figure 1).

(3) A full semester research or design project connected with nanoscience or nanotechnology.

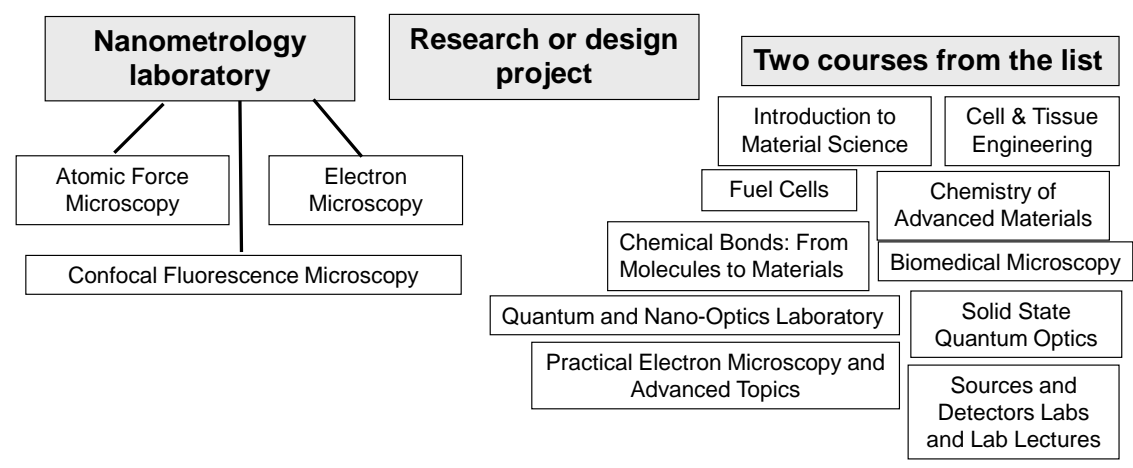

Figure 1. Required courses for a Certificate in Nanoengineering and Nanoscience. 
Here is one student's letter to the organizer of this program (Lukishova) after this student's awarding the Certificate: "After taking your nano-metrology courses, I have decided to pursue a Ph.D. in photonics \& nanoscale optical devices. I loved that I learned, and I want to do it for the rest".

The Institute of Optics administration, Dean's office of Hajim School of Engineering and Applied Sciences, and the Laboratory for Laser Energetics of the UR supported this program financially (paying URNano Center fees, materials' cost and adjunct instructors' salaries).

\subsection{Required Nanometrology Laboratory class (OPT 254/PHY371)}

The new required for the Certificate Nanometrology Laboratory class became very popular: although we extended a maximum number of students in this class from 6 to 8 students, in Spring 2017, in addition to 8 students in the class, we had already 4 more students on a waiting list for the next year. This unique class without teaching assistants (instructors, experts in the fields, spend the whole lab time with the students instructing them how to work on very expensive equipment. This course contains three modules taught by 3 instructors: (1) electron microscopy (SEM and TEM) - McIntyre, (2) optical microscopy (wide-field and confocal fluorescence microscopy of single nanoemitters) and nanoobjects/nanoengineering - Lukishova, (3) atomic force microscopy (AFM) (taught by Papernov at the Optical Materials Lab of the Laboratory for Laser Energetics (LLE), unique UR facility with a laser system for inertial confinement fusion).

During a semester students have 3 exams in each module separately and should submit individual lab reports for each module. Figure 2 shows four from eight UR students (they were divided into two groups for the labs) and their

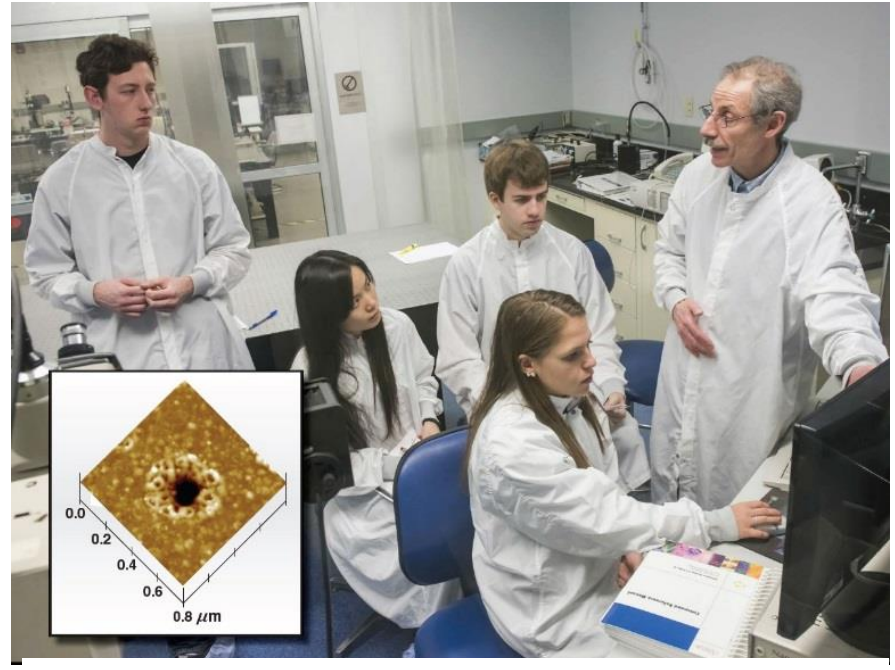

Figure 2. LLE scientist Dr. S. Papernov is shown here with four of the eight students during a teaching session on AFM. The AFM instruction is part of OPT 254, a required undergraduate laboratory course offered through the Institute of Optics (reproduced with permission from LLE). instructor from LLE near an AFM located at LLE. Students studied a laser-damage spot (see inset).

\subsection{Undergraduate research projects and some other classes of the Certificate program}

In addition to a Nanometrology Laboratory class, two other requirements to the students participating in a Certificate program are: (1) two other classes on students' selection that include nanoscience topics; (2) research or design project in nanoscience and nanoengineering.

Figure 3 shows a senior student awarded the Certificate near the confocal fluorescence microscope with a sample of nanodiamonds with NV-color centers for single-photon source applications (Lukishova's lab, Optics). It was the subject of his research for the Certificate.

Some other students' research projects are:

- Development of micro-arrayed label-free biosensor using theoretical \& experimental analysis of arrayed imaging reflectometry (Miller's lab, Biomedical Engineering).

- Adaptive optics scanning light ophthalmoscope (nanoscale measurements) and how it can effect a disease called Achromatopsia (Merigan's lab, Center for Visual Science).

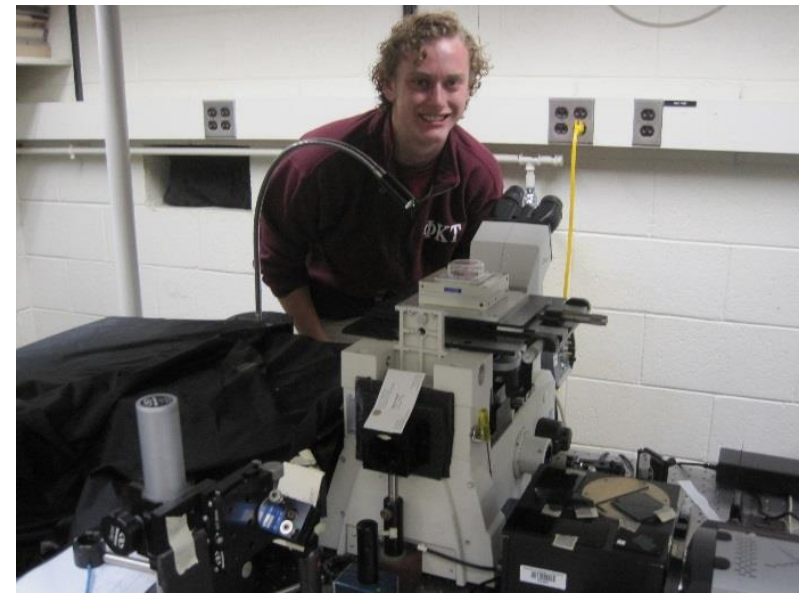

Figure 3. Senior Andrew Kruse is working on his research project on nanodiamonds applications to the single-photon sources. He was awarded the Certificate in 2016.

- The effects of nanoparticle-mediated siRNA delivery on human mesenchymal stem cell proliferation (Benoit's lab, Biomedical Engineering). 
- RF Bias-assisted sputtering of Silicon Nitride thin films on Silicon wafers (Cardenas' lab, Optics).

- A polarization sensitive atomically-thin photodiode (Vamivakas' lab, Optics).

- Quantum-enabled super-resolution microscopy (Vamivakas' lab).

The most popular class from two elective classes for the Certificate is OPT 253 Quantum and Nano-Optics Laboratory (Lukishova) ( http://www.optics.rochester.edu/workgroups/lukishova/QuantumOpticsLab/ ). Figure 4 shows students of this class (20 students in Fall 2016) on the lecture on nanophotonics and nanoplasmonics (left) and near a portative AFM preparing to measure topography of nanodiamonds (right). In addition to students' experience with AFM, nano-optics part of this class consists of two laboratory experiments: (1) confocal fluorescence microscopy and spectroscopy of singleemitter nanocrystals and (2) photon antibunching measurements from single nanoemitters using a Hanbury Brown and Twiss intensity interferometer. These two labs are carried out on a research setup (Lukishova's group) on single-photon generation and characterization. Students are working in real research environment on expensive single-photon counting equipment that easy to destroy: single-photon counting Si avalanche photodiode modules and electron-multiplying CCD cameras sensitive to single photons. Real research samples are used for teaching and students sometimes obtain the results during a lab class that later were included to the conference proceedings, for instance, Figure 4 shows the processed results from students'lab of 2015 that were reported in Ref. [2]: confocal microscope fluorescence imaging of gold bowtie nanoantenna arrays and CdSeTe colloidal nanocrystal quantum dots (NQD) within a gaps of bowtie arms (Figure 4, left), time traces of NQD fluorescence blinking (Figure 4, center) and photon antibunching from NQDs within bowtie nanoantenna gaps (Figure 4, right) with a dip indicating nonclassical behavior, antibunching (separation of all photons in time).
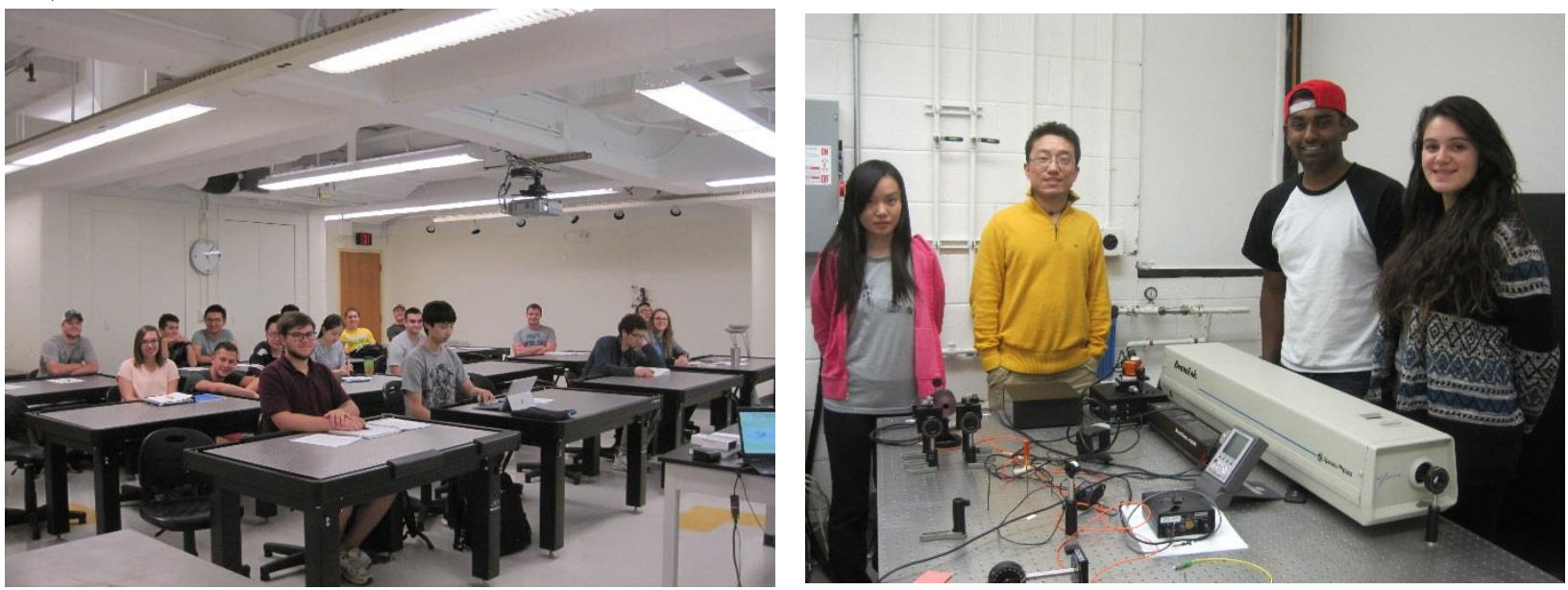

Figure 4. LEFT: Students on the lecture on nanophotonics and nanoplasmonics (OPT 253 class). RIGHT: Optics and biomedical engineering students preparing to AFM measurements of topography of nanodiamonds (OPT 253 class).
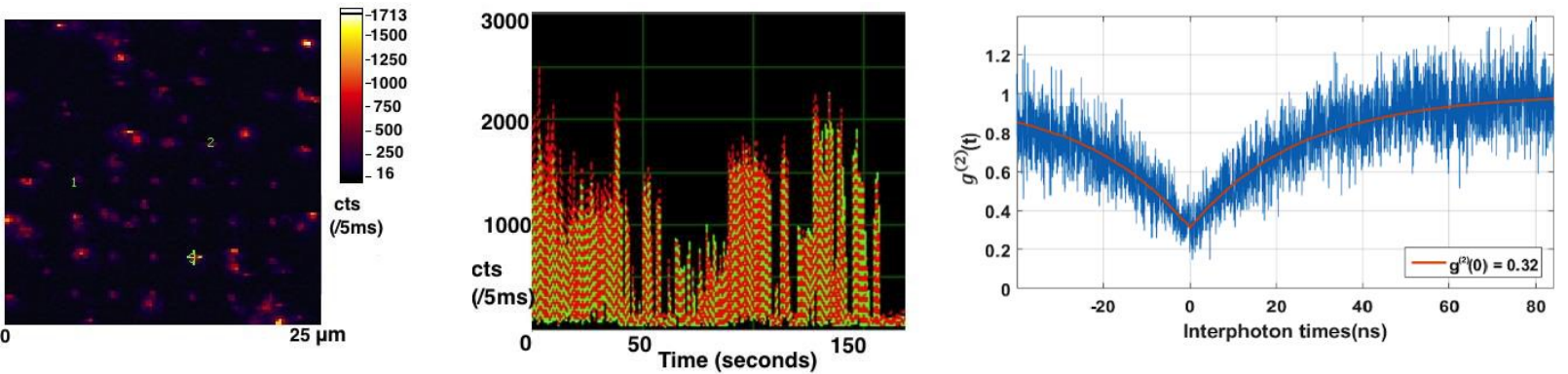

Figure 5. From the results obtained in a lab class of OPT 253. LEFT: Confocal microscope fluorescence image of a gold bowtie nanoantenna array and spin-coated NQDs (cross shows a position of NQD with photon antibunching). CENTER: Time trace with NQD blinking within a bowtie nanoantenna (photon antibunching of this NQD was recorded). RIGHT: Typical antibunching curve of NQD fluorescence within bowtie nanoantenna (dip in the center of the second order correlation function $\mathrm{g}^{(2)}(\mathrm{t})$, where $\mathrm{t}$ is interphoton time. For NQD within several different bowtie nanoantennas $\mathrm{g}^{(2)}(0)$ varied from 0.15 to 0.7 (from processing of OPT 253 students' results). 
A graduate version of this class is a required class for Master students who select their specialty Nano- and Integrated Photonics. Although both undergraduate and graduate students carry out the labs in the same groups, graduate students in addition to all lab reports and two exams should submit their individual essay on single (antibunched) and entangled photon sources and deliver a final individual Power Point slides presentation about the content of four labs of this class.

Because of lack of space in a Curriculum for new classes, we modified a required class for optics and optical engineering majors OPT 204 Sources and Detectors Labs and Lab Lectures (Lukishova) by including content on nanoscience and nanotechnology. Now OPT 204 is listed as a possible elective class for the Certificate. To satisfy Certificate program requirements we included lab experiments with CdSe NQD solutions as bright fluorescence sources with calculation of their sizes from spectral measurements and on measurements in a spectrophotometer with integrating sphere a reflectivity of liquid crystal photonics bandgap material showing photonic stop band. Students also measured photon statistics of laser and pseudothermal light sources with a single-photon counting detector, widely used in nanoscience. On two lectureworkshops on Lasers and Nonclassical Light Sources nanolasers and nanophotonic advances for antibunched photon sources were discussed.

\subsection{Undergraduate students' training during summer 2016}

In summer 2016, nine Rochester optics undergraduate students took part in educational events in Moscow and St. Petersburg as part of the U.S.-Russian collaboration (see Ref. [3] and paper [4] of this ETOP proceedings). In Moscow, Rochester students were immersed for five days in cutting-edge research, technologies and ideas on offer from Russian, European and U.S. scientists at the National Research Nuclear University MEPhI International School on Optics and Laser Physics. This experience also included tours of a MEPhI's Center on Nanotechnology with its clean-room facility for electronics components and its Nano-bioengineering laboratory. They also visited laboratories of Lebedev Physical and General Physics Institutes of the Russian Academy of Sciences, for instance, facilities for growing diamond thin films and nanocrystals with single defect centers and learned about cutting edge research on colloidal semiconductor nanocrystal quantum dots. After one week in Moscow students moved to St-Petersburg for a research work on photonics and nanophotonics in the scientific laboratories of the International Institute "Photonics and Optoinformatics" of ITMO University [5]. At the end each UR student delivered 15-min talks about their research and obtained the Certificates of this School on Photonics. These collaboration with Russian institutions was outlined in the magazine of the Optical Society OSA Optics and Photonics News [3]. Here are some students' comments after their MEPhI visit:

- ...The lab tour I like the most of all would be the tour of the laboratories of MEPhI scientific educational center "Nanotechnologies". It is my first time attending a lab focusing on nanotechnology...

- ....Some of my favorite lectures are "Carbon Photonics" given by Prof. Vitali I. Konov, "Organic Nanophotonics" given by Prof. Alexei Vitukhnovsky and "Laser Printing of sensing plasmonic nanostructures" given by Dr. Yuri N. Kulchin. My favorite labs are laser centers and lab for the nanotechnologies. I was really fascinated by the two different lithography systems of laser lithography and electron beam lithography during the visit to the nanotechnology center in MEPhI...

Now several students of this trip are working towards the Certificate.

\section{TRAINING MONROE COMMUNITY COLLEGE STUDENTS AT THE UR}

Described in this section joint efforts of UR and MCC can serve a reproducible model of collaboration between a university with strong programs in science and technology and modern experimental facilities, and a nearby, two-year community college. One challenge facing Physics courses at community colleges is the lack of student activities centered on modern experiments. The lab of the typical course consists predominately of replicating nearly 100-year-old experiments (photoelectric effect, hydrogen spectroscopy, etc.). Addition of a state-of-the-art lab experience makes the course truly modern and provides a strong motivating force for future STEM study. Experience at the URNano Center not only enhanced the laboratory portion of MCC's Modern Physics course, helping to prepare MCC students for employment, but also made easier the transition for students enrolled in the $2+2$ transfer option. In addition, Liberal Arts students taking the MCC "Physics for Non-majors" course also participated in the project.

NSF NUE grant permitted to continue UR-MCC collaboration that was supported earlier by previous NSF grants (see ETOP paper [1]). From 2009 to 2013, 90 MCC students carried out two, three-hour labs (single photon interference and entangled photons and Bell's inequalities) in two-week stints at the UR. In years 2014-2015, other 52 MCC students with NUE grant support carried out two labs at the UR described in this section. P.D. D'Alessandris led this project from MCC. 
After the labs, MCC students answered both conceptual and quantitative questions about the experiments. Evaluation showed a very high percent of correct answers. Formative evaluation showed overwhelmingly positive MCC student response to this experience.

\subsection{Atomic force microscopy of nanodiamonds}

52 MCC students were trained in Spring 2014-2015 on a compact AFM, EasyScan 2, Nanosurf (Lukishova's lab). During 3-hour lab they measured a topography of nanodiamonds. Figure 6, left shows Monroe Community College students near an AFM located on a vibration isolation optical table. A magnified picture of AFM is shown as an insert. Figure 6, right shows a typical, unprocessed AFM micrograph of nanodiamonds obtained by this AFM.
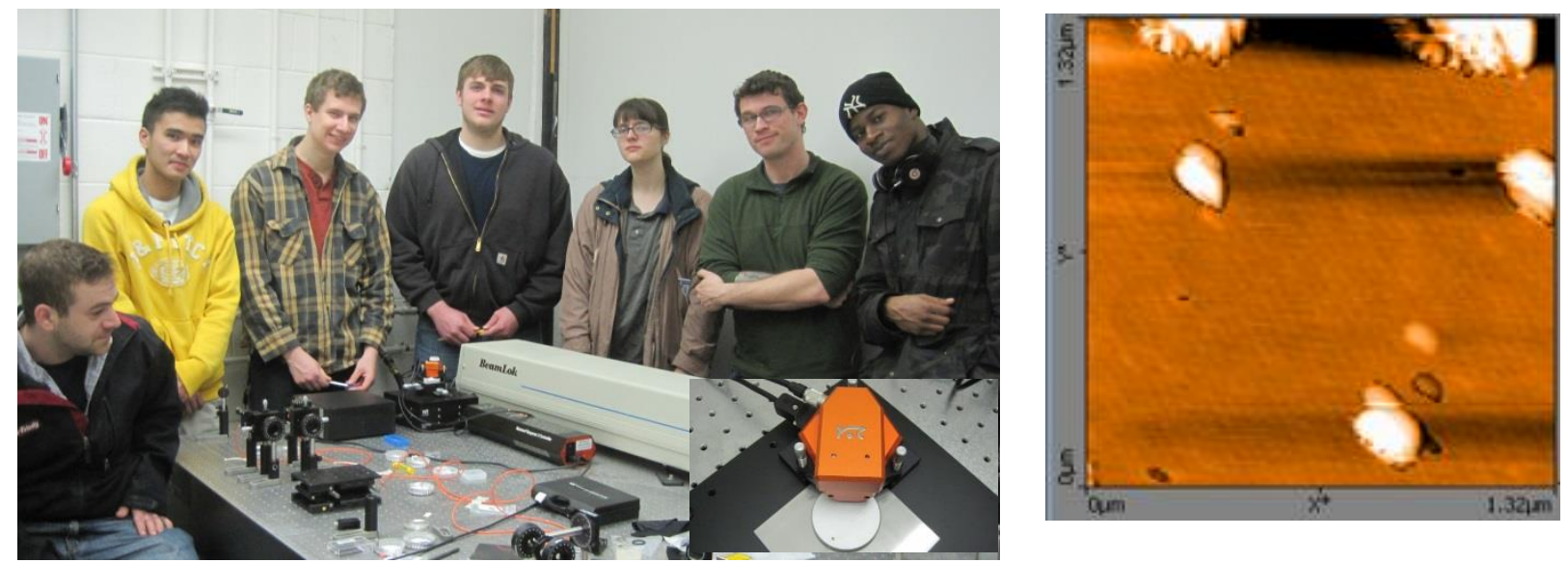

Figure 6. LEFT: Monroe Community College students during an AFM lab. (A small orange-color AFM and its controller (black) are located at the left side of the picture). INSERT: A magnified picture of an AFM. RIGHT: Typical unprocessed AFM micrograph of nanodiamonds.

\subsection{UR NanoCenter, clean-room facility and a photolithography lab}

In a collaboration with the UR NanoCenter (McIntyre) a 3-hour lab on photolithography in a clean room was developed for MCC students. Several groups of MCC students prepared the MCC and UR logos using a photolithography and vacuum deposition of a metal (Figure 7, left). Before the lab students were trained in working in a clean-room facility passing through the quiz. Figure 7, right shows two groups of MCC students before entering a clean room.

MCC students also participated in the tour of the whole University of Rochester NanoCenter (Figures 8-9) with the lecture (McIntyre) about its equipment including electron beam microscopes (Figure 9, top left).
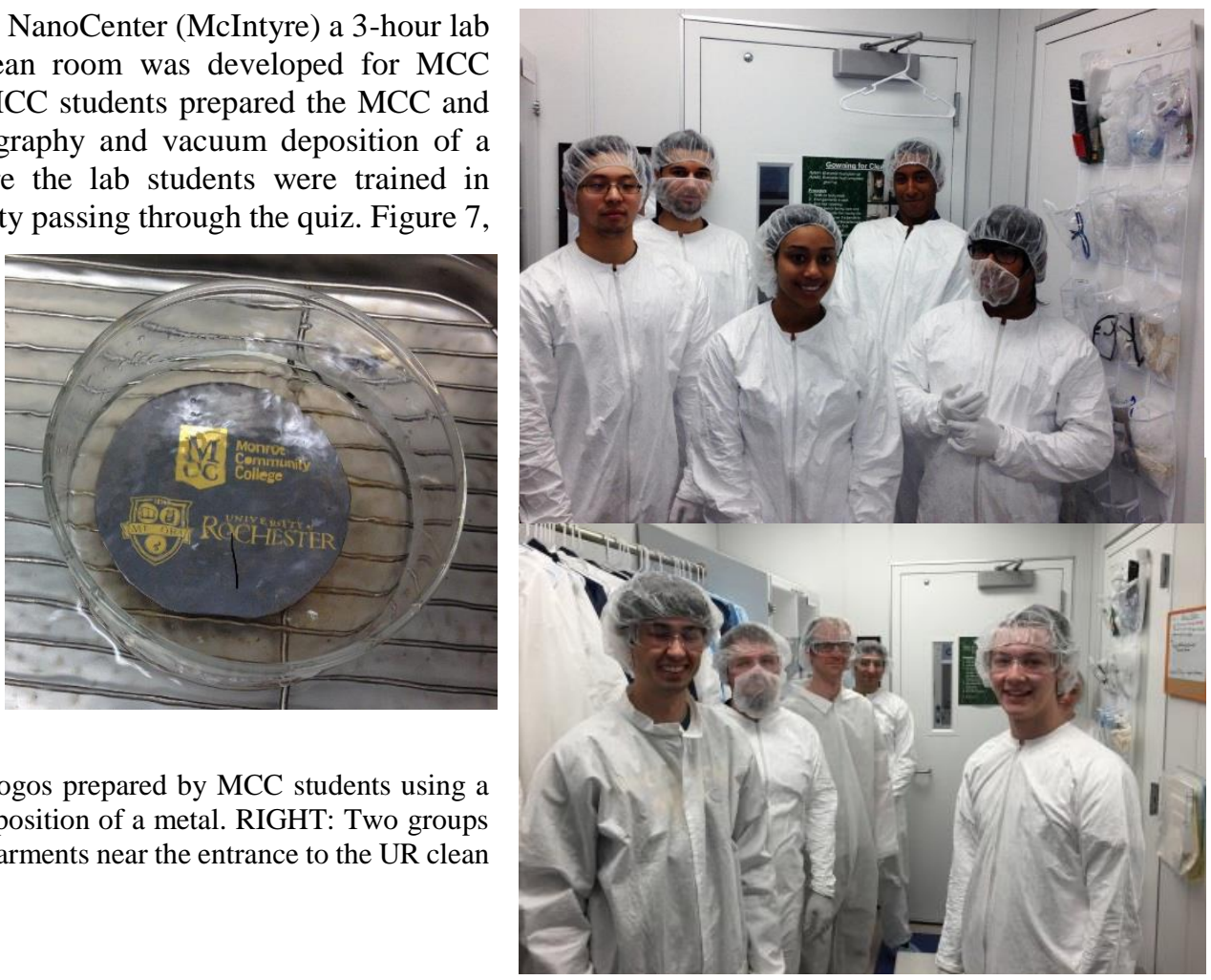

Figure 7. LEFT: MCC and UR logos prepared by MCC students using a photolithography and vacuum deposition of a metal. RIGHT: Two groups of MCC tudents in clean-room garments near the entrance to the UR clean room. 


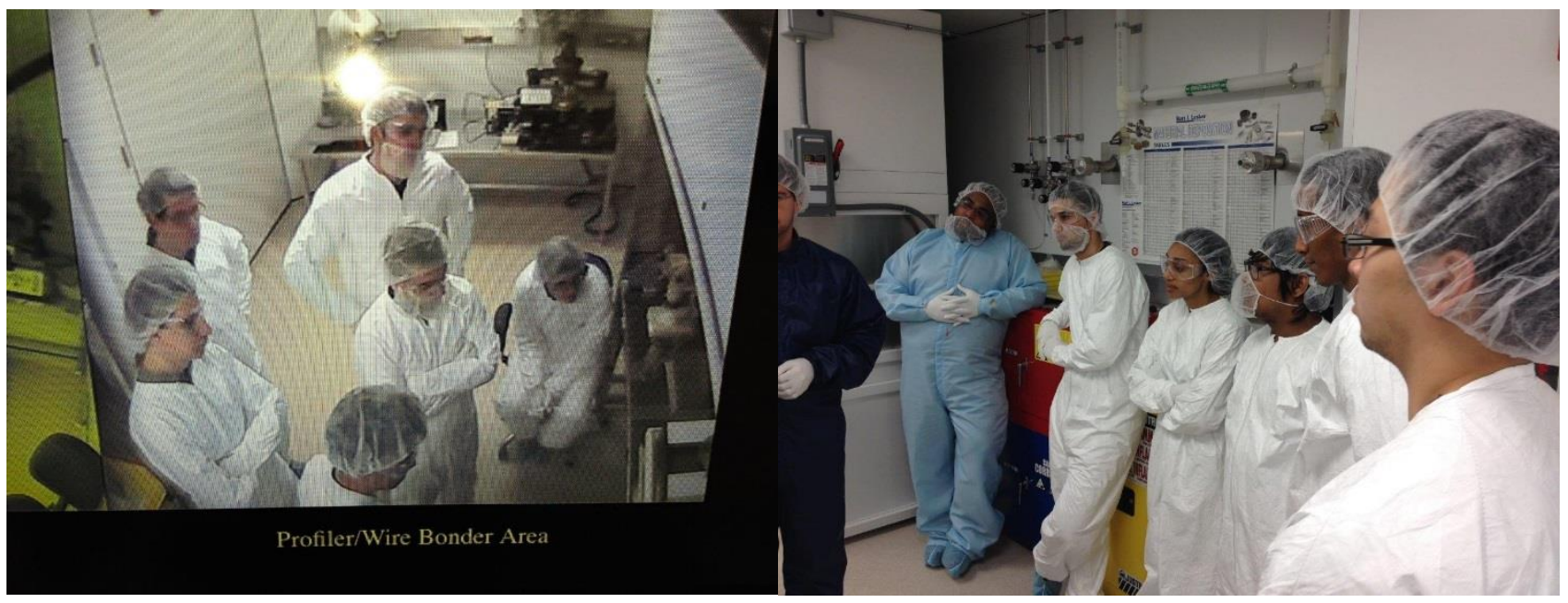

Figure 8. MCC students in the URNano Center clean room during sample preparation.

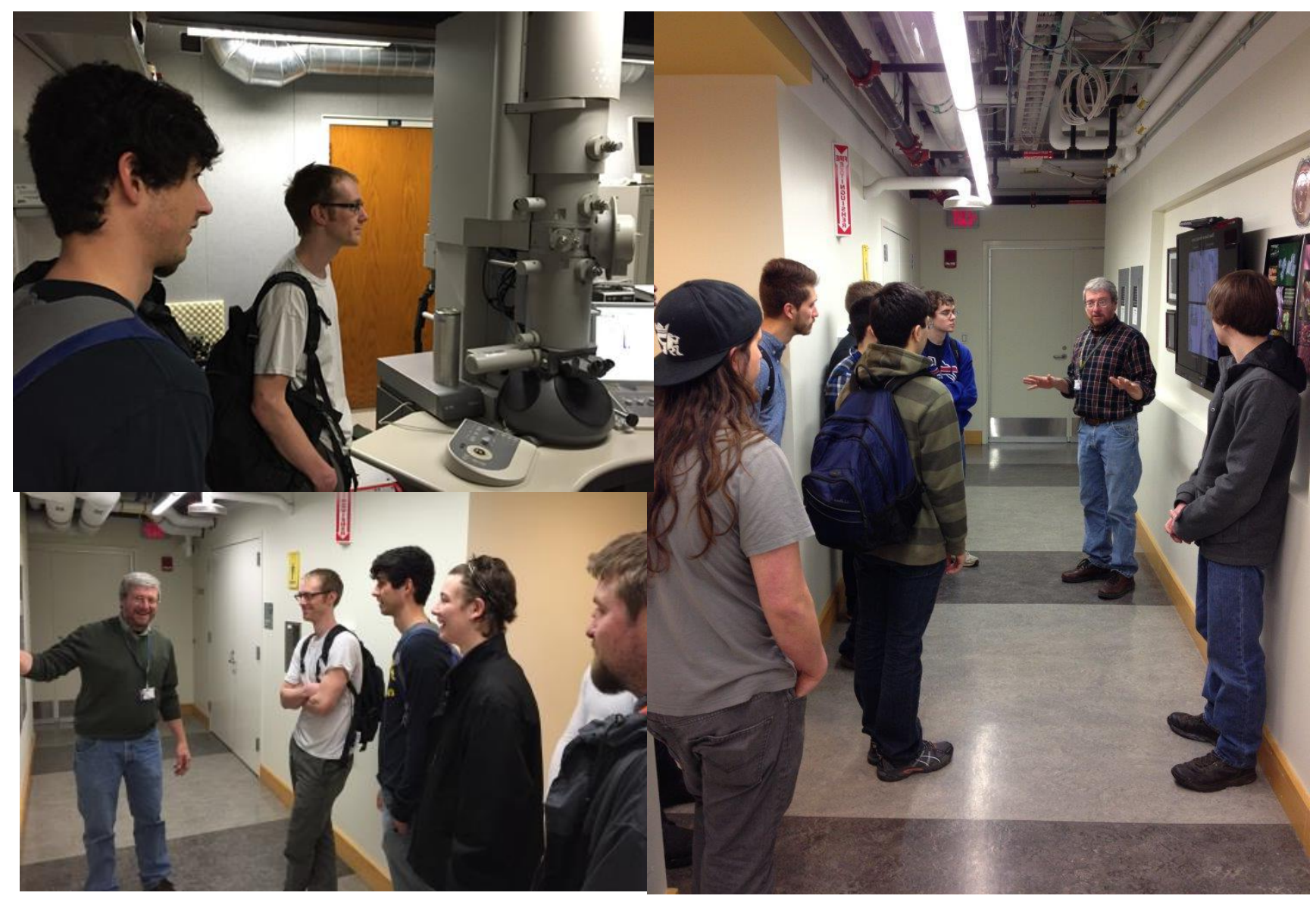

Figure 9. MCC students during lecture-demonstration of B. McIntyre of URNano Center facilities. 


\section{INTRODUCING "MINI-LABS" ON NANOMATERIALS AND NANOSTRUCTURES INTO EXISTING CLASSES}

This section describes examples of two "mini-labs" that were introduced in classes OPT 204 Sources and Detectors Labs and Lab Lectures (Lukishova) and OPT 253/OPT 453 Quantum and Nano-Optics Laboratory.

\subsection{Measuring spectra of colloidal nanocrystal quantum dots and evaluating their sizes from spectral measurements using the time-independent Schrödinger equation}

We took the idea from the NanoSys company which sells some teaching materials. MCC (D'Alessandris) bought from this company a kit with NQDs for this lab. UR used NQDs with different fluorescence wavelengths from the UR Chemistry Department (Krauss).

Electronic and optical characteristics of a nanocrystal quantum dot (NQD) are closely related to its size and shape. For example, the band gap in a NQD which determines the frequency range of emitted light is inversely related to its size. In fluorescence applications the frequency of emitted light increases as the size of the quantum dot decreases. Consequently, the color of emitted light shifts from red to blue when the size of the quantum dot is made smaller.

In order for the NQD to produce photoluminescence, an exciton (electron and hole) must be created. When the electron and hole recombine, they create the photoluminescence. In this lab experiments the exciton is created by the absorption of UV or blue light. The solutions of NQDs (prepared by Krauss' group, UR) were excited either by a blue light of the laser pointer or by a special UV source shown in Figure 10, left. Students wear UV protective goggles during this experiment. NQD fluorescence was observed by the Ocean Optics spectrometer.
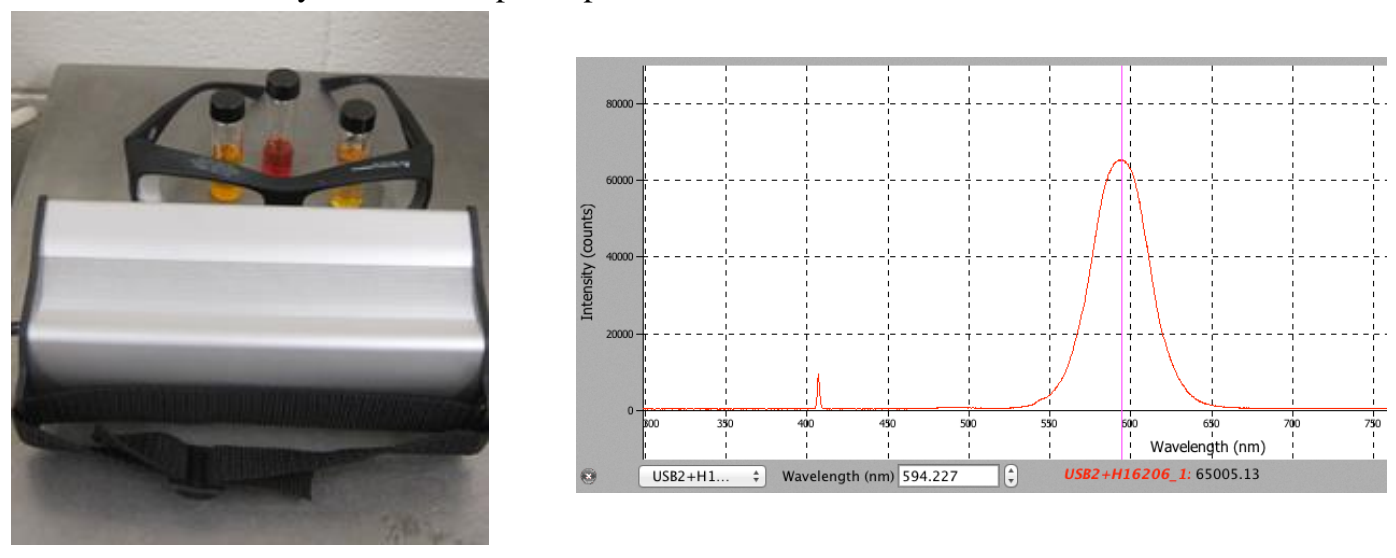

Figure 10. LEFT: Three solutions of NQDs and UV source for excitation of NQD fluorescence. RIGHT: Typical fluorescence spectrum of NQD.

To calculate the size of NQD from their maximum fluorescence wavelength, NQD should be modeled as a quantum particle in the box with discrete energy levels. From the solution of the time-independent Schrödinger equation for the particle in the box (infinite potential well) in three-dimensional case, one can find dependence of energy $E$ of the NQD on the radius $\mathrm{R}$ that can be expressed by the following equation:

$$
\mathrm{E}=\frac{\hbar^{2} \pi^{2}}{2 \mathrm{~m}_{\mathrm{e}} \mathrm{R}^{2}}+\frac{\hbar^{2} \pi^{2}}{2 \mathrm{~m}_{\mathrm{h}} \mathrm{R}^{2}}+E_{\mathrm{g}} \quad \begin{aligned}
& \mathrm{E}_{\mathrm{g}}=2.15 \times 10^{-19} \mathrm{~J} \\
& \mathrm{~m}_{\mathrm{e}}=7.29 \times 10^{-32} \mathrm{~kg} \\
& \mathrm{~m}_{\mathrm{h}}=5.47 \times 10^{-31} \mathrm{~kg}
\end{aligned}
$$

$E_{\mathrm{g}}$ is the energy that comes from the energy of the semiconductor bandgap; $\mathrm{m}_{\mathrm{e}}$ and $\mathrm{m}_{\mathrm{h}}$ are effective masses of electron and hole. Using these equations you can easy calculate the sizes of quantum dots from spectral measurements of their fluorescence. 


\subsection{Cholesteric liquid crystal photonic bandgap materials: fabrication and measuring their reflectivity along a stopband}

Planar-aligned cholesteric liquid crystal (CLC) structures exhibit a 1-D photonic bandgaps (light at some frequency band is reflected) for the handedness of circularly-polarized light where the electric field vector follows the rotation of the CLC molecular director (see Figure 11, left below). The stop band is centered at wavelength $\lambda_{o}=p\left(n_{e}+n_{o}\right) / 2$, where $p$ is the pitch of the CLC spiral structure, and $n_{e}$ and $n_{o}$ are extraordinary and ordinary refractive indexes. The bandwidth of the transmission stop band is given by $\Delta \lambda \sim \mathrm{p}\left(\mathrm{n}_{\mathrm{e}}-\mathrm{n}_{\mathrm{o}}\right)$.

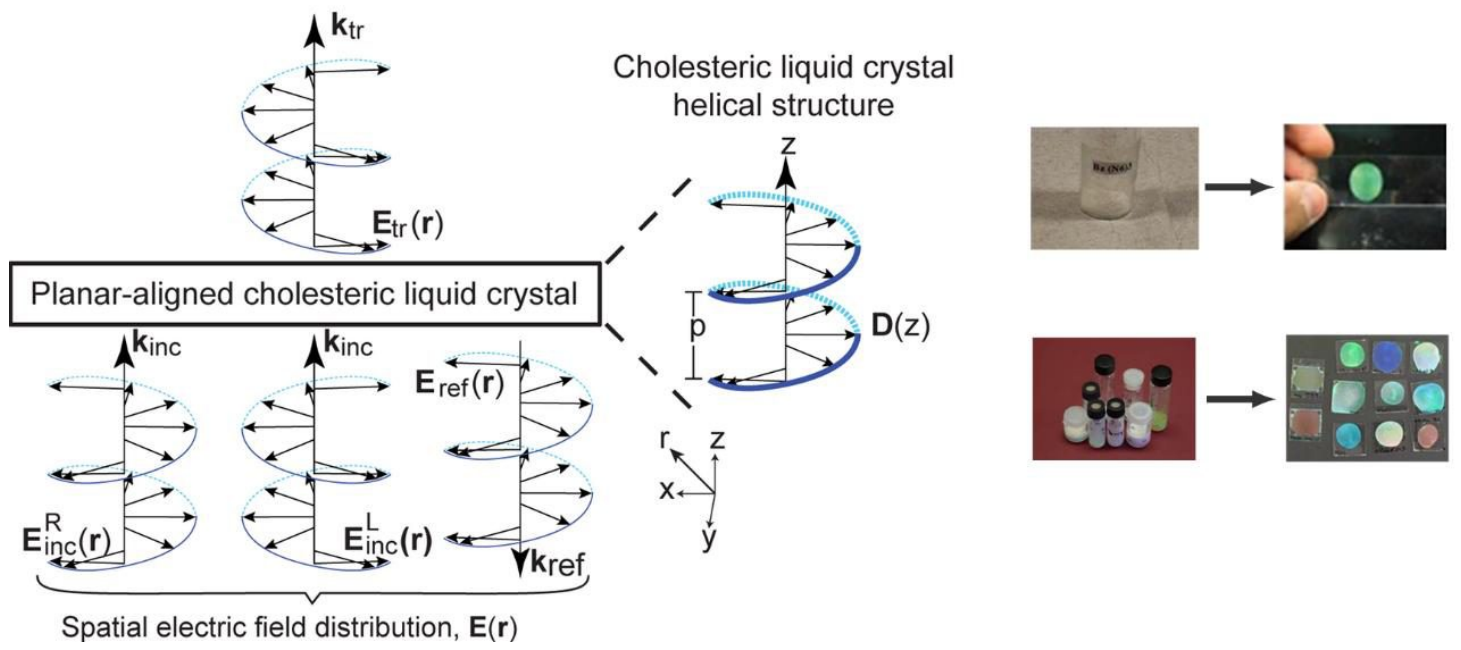

Figure 11. LEFT: Circular polarized light propagation in a 1-D photonic bandgap CLC structure. RIGHT: Prepared photonic bandgap CLC structures from a powder oligomer (top left) and monomeric LCs (bottom left).

These structures are simplest in preparation of photonic bandgap material. They can be prepared either from a cholesteric oligomeric powder (Figure 11 , right top) by heating the powder between two cover glass slips on a hotplate $\left(\sim 70-100^{\circ} \mathrm{C}\right)$ to the liquid state. With the liquid state of a cholesteric material the substrates should be sheared for planar alignment of liquid crystal molecules and cooled for a glassy (solid) state of a liquid crystal preserving an alignment. Nice color appears from an initial powder oligomer (see Figure 11, right) as a manifestation of a photonic bandgap (selective reflection) of the structure. Using a monomeric (liquid form) liquid crystal preparation of a photonic bandgap takes only 1 minute: the drop of a monomeric cholesteric liquid crystal should be placed between two glass slips, and after their shearing, a uniform color from an initial "milky" liquid crystal will appear. Figure 12 shows biomedical engineering students of OPT 253 class with prepared samples of a liquid crystal photonic bandgap material for a room-temperature single-

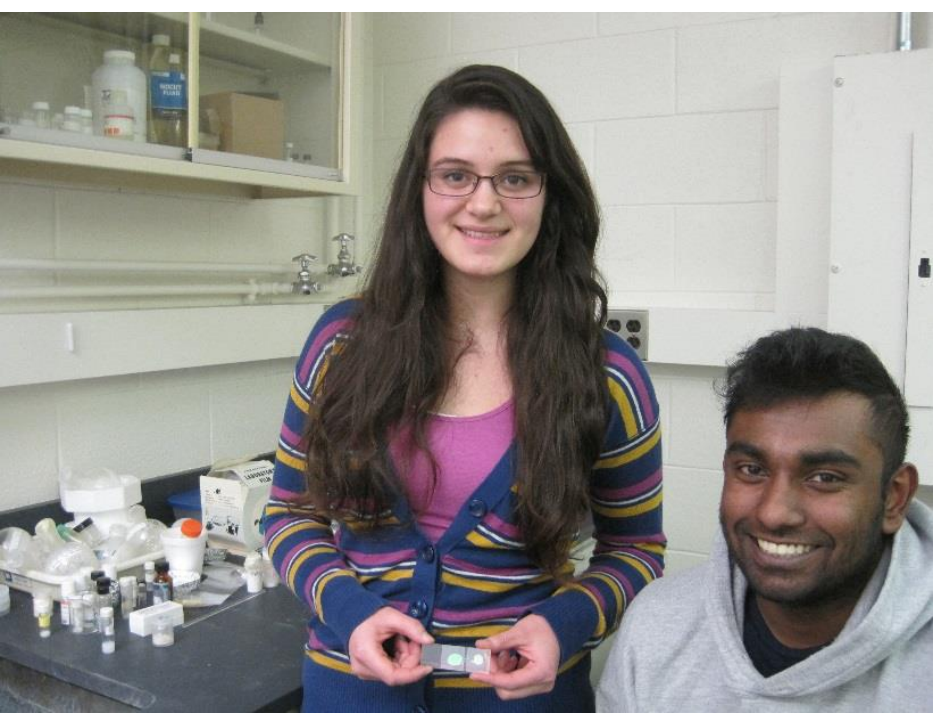

Figure 12. Biomedical engineering students from the OPT 253 class with prepared sample of cholesteric liquid crystal photonic bandgap material for a room-temperature single-photon source [6]. 
This lab was very popular among middle and high school students and their parents during the Institute of Optics Family Day of showing Optics lab experiments to children.

Students of OPT 204 measured reflectivity of cholesteric liquid crystal material on their lab on spectroscopy of different light sources and radiometry using a spectrophotometer Konica Minolta CM-3700 A with an integrating sphere (Figure 13, left). Insert (left) shows the samples of photonic bandgap CLC structures. Spectral dependence of reflectivity of one of the samples is shown in Figure 13, right (spectrophotometer user interface).
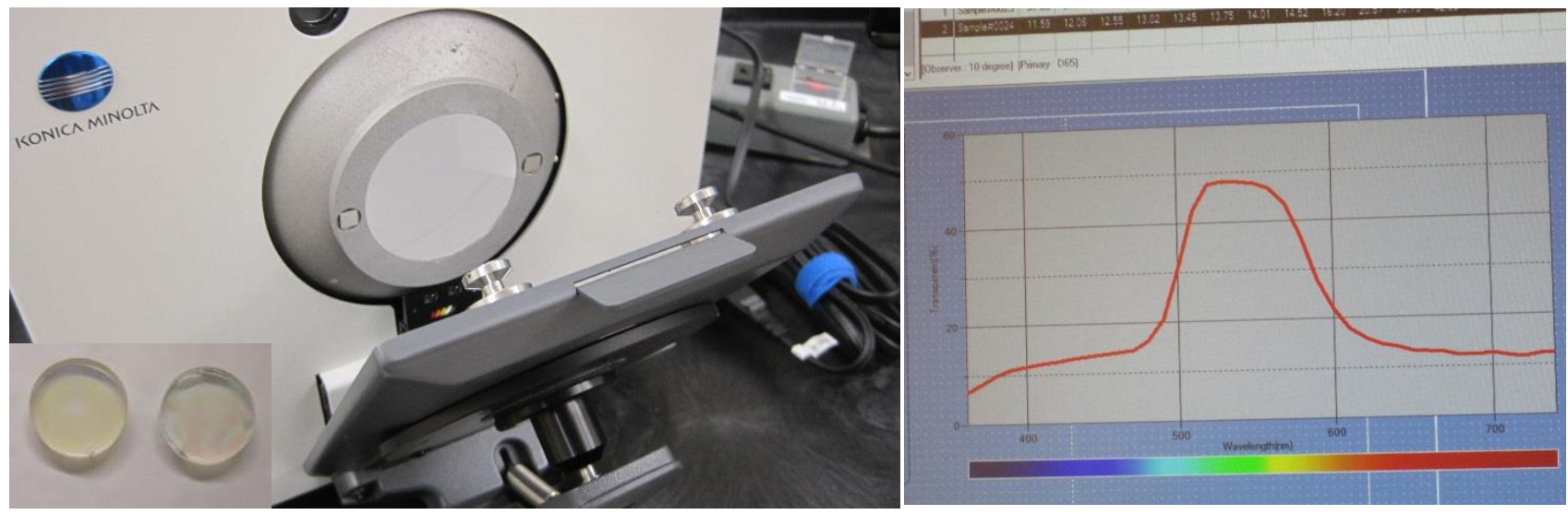

Figure 13. LEFT: Integrating sphere inside a spectrophotometer. CLC samples between two glass substrates are shown in insert. RIGHT: A selective reflection curve (spectral dependence of reflectivity) of a photonic bandgap CLC structure.

\section{PEDAGOGICAL RESEARCH ON STUDENTS’ LEARNING}

How do we know whether students are learning? What did they learn? How well did they learn? What are their learning outcomes? To answer these questions we used two evaluation-methodology approaches:

- A qualitative one (individual and group interviews, focus group technique, observations, end-of-course evaluations, student surveys, content analysis of student reports, etc.), and

- A quantitative one (grades, questionnaires, etc.).

In pedagogical research, we collaborated with the external evaluator J. Zawicki (State College at Buffalo). For NSF grant reports external evaluation of the project's success is mandatory.

Here is one example from the spring 2014-2015 with MCC students who completed three assessment measures during the spring 2014 semester. They completed both pre- and post-experience using two lab experiments: a Cleanroom Access Quiz, and an Atomic Force Microscope Quiz. The pre-assessments were delivered prior to formal instruction; postassessment measures were administered following the labs. The data provided evidence that MCC students were benefitting from the laboratory experiences at the UR. The pre- and post-assessment data indicated substantial growth in student understanding.

\section{CONCLUSION}

This paper describes a realization of nanotechnology/nanoscience undergraduate education program at the University of Rochester led by Integrated Nanosystems Center (directed by N. Bigelow). The program was supported by the NSF educational grant (2014-2016). The main achievements of this programs are:

- creating a coherent educational program at the UR on the Certificate for Nanoscience and Nanoengineering being issued to 15 students;

- creating a transformable model of collaboration in nanotechnology between a university with state-of-the-art, expensive experimental facilities, and a nearby, two-year community college on the example of Monroe Community College (52 MCC students carried out two 3-hour labs on nanotechnology at the UR);

- developing reproducible hand-on experiments ("mini-labs"), learning materials and pedagogical methods to educate students with diverse backgrounds, including freshmen and non-STEM-major CC students. 


\section{ACKNOWLEDGEMENTS}

We acknowledge contributions of B. McIntyre and S. Papernov to a Nanometrology lab class. We thank a Laboratory for Laser Energetics for supporting an AFM lab class, Hajim Schhol of Engineering and Applied Sciences Dean's office, the Institute of Optics administration for support of a Nanometrology lab class. This project was supported by the NSF educational grant EEC-1343673 "NUE: Development of Multidisciplinary Nanotechnology Undergraduate Education Program at the University of Rochester Integrated Nanosystems Center".

\section{REFERENCES}

[1] Lukishova, S.G., "Quantum optics and nano-optics teaching laboratory for the undergraduate curriculum: teaching quantum mechanics and nano-physics with photon counting instrumentation", Paper 100-189, 14th International Conference on Education and Training in Optics \& Photonics (ETOP), May 29-31, 2017, Hangzhou, China.

[2] Lukishova, S.G., Mihaylova, D., Zhu, H., Liapis, A., Boyd, R.W., "Plasmonic bowtie nanoantennas with nanocrystal quantum dots for single-photon source applications", Frontiers in Optics 2016, OSA Technical Digest, (Optical Society of America, Rochester NY, September 17-21, 2016), paper LF2D.6, https://doi.org/10.1364/LS.2016.LF2D.6.

[3] Lukishova, S.G., Kozlov, S.A., Zavestovskaya, I.N., “A Rochester-Russia Collaboration”, Optics \& Photonics News, pp. 20-21, November 2016.

[4] Lukishova, S.G., Zavestovskaya, I.N., Zhang, X.-C., Aleshchenko, Yu.A., Konov, V.I., paper 100-190,“Launching partnership in optics and photonics education between University of Rochester and Moscow Engineering Physics Institute NRNU MEPhI", paper 100-190, the $14^{\text {th }}$ International Conference on Education and Training in Optics \& Photonics (ETOP), May 29-31, 2017, Hangzhou, China.

[5] Egorov, V., Buyanovskaya, E., Melnik, M., Gleim, A. Lukishova, S., Kozlov, S., Zhang, X.-C., "Research summer camp in photonics", paper 100-59, 14th International Conference on Education and Training in Optics \& Photonics (ETOP), May 29-31, 2017, Hangzhou, China.

[6] Lukishova, S.G., "Liquid crystals under two extremes: (1) high-power laser irradiation, and (2) single-photon level", Molecular Crystals and Liquid Crystals 559, Special Issue, 127-157 (2012). 